\title{
Successful less-invasive endoscopic treatment for bile leakage from choledochojejunostomy site using short-type single-balloon enteroscopy
}

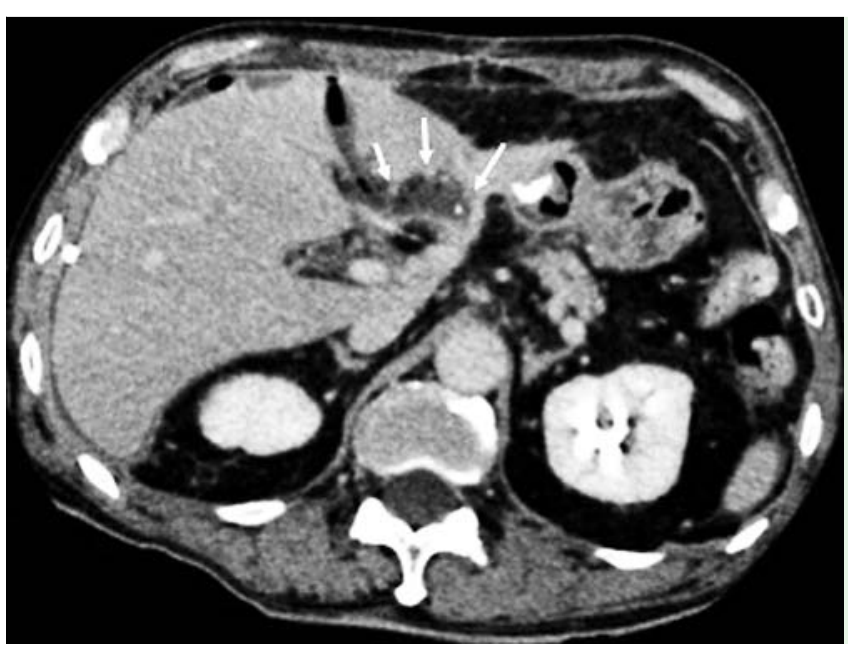

Fig. 1 Enhanced

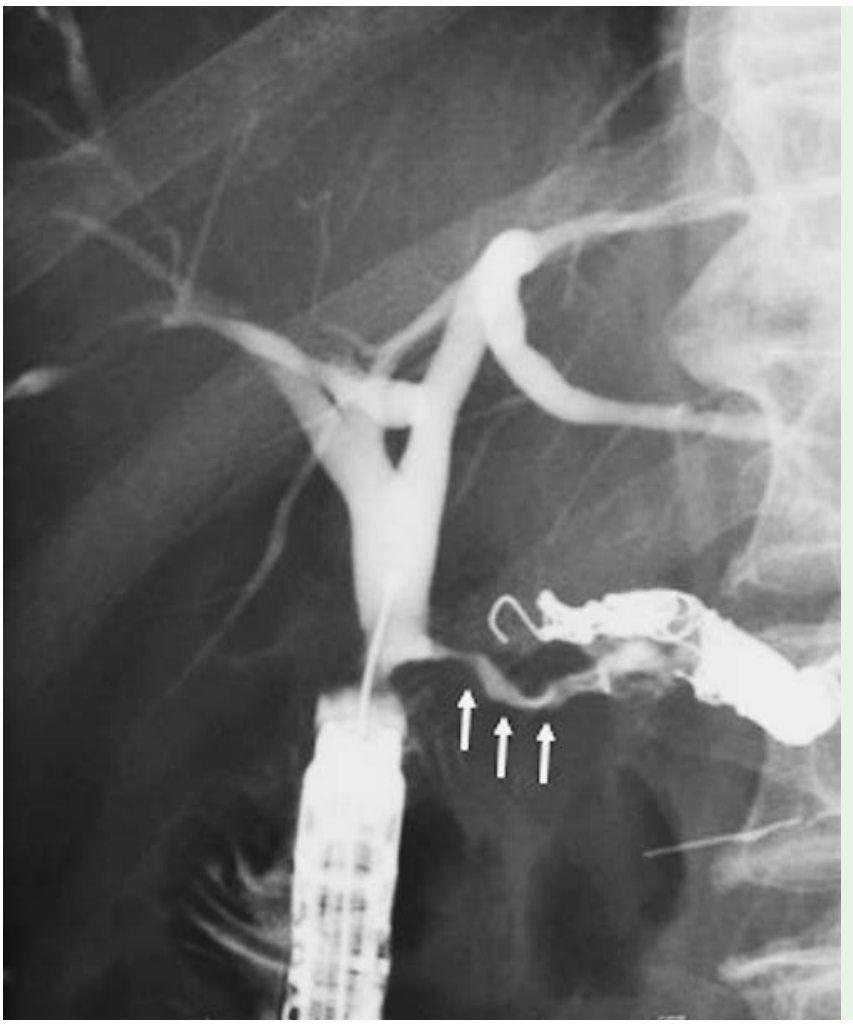
computed tomography showed abdominal fluid collection around the anastomosis (arrows).

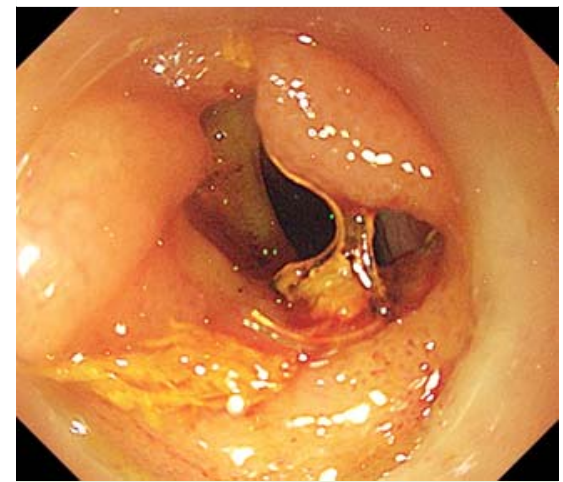

Fig. 2 Single-balloon enteroscopy revealed the choledochojejunostomy site without stenosis.

Fig. 3 Cholangiography showed bile leakage at the anastomosis (arrows).

A 76-year-old man who had undergone subtotal stomach-preserving pancreaticoduodenectomy for pancreatic head cancer 1 month previously presented with
Bile leakage from the choledochojejunostomy site is usually treated by percutaneous transhepatic biliary drainage (PTBD) or reoperation [1,2]. To date, there have been no reports on endoscopic treatment for bile leakage from the choledochojejunostomy site. Recently, balloon enteroscopy was developed and used to perform endoscopic retrograde cholan- giopancreatography (ERCP) in patients with surgically altered gastrointestinal anatomy [3-5]. To the best of our knowledge, this is the first case report of balloon enteroscopy-assisted ERCP for bile leakage from the choledochojejunostomy site following transcatheter arterial embolization (TAE). melena. A pseudoaneurysm was detected at the distal part of the gastroduodenal artery on computed tomography (CT), which was treated by TAE. Three days after the procedure, the patient experienced abdominal pain and fever, and an abdominal abscess was found on CT ( $\bullet$ Fig. 1). The cause of the abscess was thought to be anastomotic leakage, which was possibly related to the impairment of blood flow to the bile duct following TAE. PTBD was considered to be difficult because there was no dilation of the bile duct. Therefore, ERCP assisted by prototype, short-type, single-balloon enteroscopy (short-SBE: SIF-Y0015; Olympus Medical Systems, Tokyo, Japan) was attempted.

The choledochojejunostomy site could be identified using short-SBE ( $\bullet$ Fig. 2 ), and cholangiography showed bile leakage at the anastomosis ( $\bullet$ Fig. 3). A straight-type 5-Fr nasobiliary drainage tube (Gadelius Medical KK, Tokyo, Japan) was placed on the left hepatic duct without any adverse events ( Video 1$)$.

One week after the procedure, the nasobiliary tube was removed after confirming the absence of bile leakage by cholangiography ( $\bullet$ Fig.4). A 7-Fr pigtail stent (Gadelius Medical KK) was then placed to B3 using short-SBE. The biliary stent was removed after 1 month. At the 3-month 


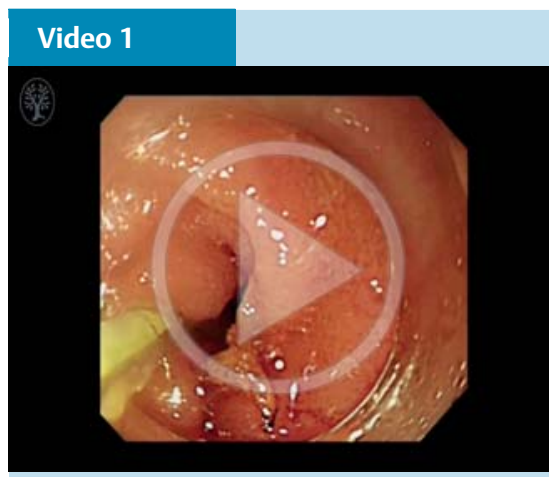

A short-type single-balloon enteroscope was inserted into a surgically altered gastrointestinal tract, and the choledochojejunostomy site was identified. The anastomosis was observed endoscopically and showed no stenosis. Thereafter, a catheter was inserted into the bile duct and cholangiography was performed. Bile leakage at the anastomosis was confirmed under fluoroscopic guidance. Finally, a nasobiliary drainage tube was placed in the left hepatic duct.

follow-up, the patient had experienced no further episodes such as recurrence of bile leakage or anastomotic stenosis. Balloon enteroscopy-assisted ERCP was found to be an effective and less-invasive treatment for bile leakage from the choledochojejunostomy site following TAE.

Endoscopy_UCTN_Code_TTT_1AR_2AG

Competing interests: None

\section{Kazumasa Nagai, Kei Yane, Akio Katanuma, Kuniyuki Takahashi, Toshifumi Kin, Hajime Yamazaki, Hiroyuki Maguchi}

Center for Gastroenterology, Teine-Keijinkai Hospital, Sapporo, Japan

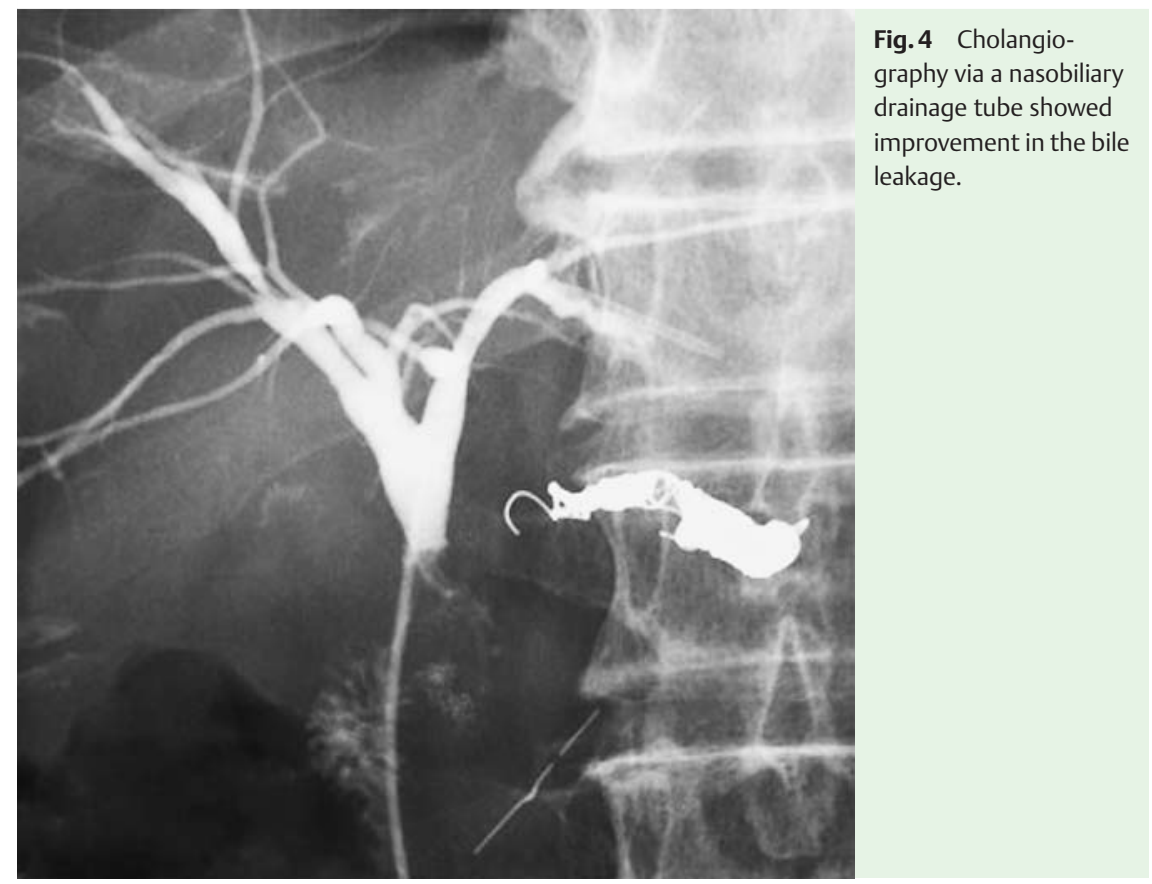

\section{Acknowledgment $\nabla$}

We thank Dr. Edward Barroga, Associate Professor and Senior Editor of Tokyo Medical University for editing the manuscript.

\section{References}

1 Li SQ Liang $L J$, Peng BG et al. Bile leakage after hepatectomy for hepatolithiasis: risk factors and management. Surgery 2007; 141: $340-345$

2 De Castro SM, Kuhlmann KF, Busch OR et al. Incidence and management of biliary leakage after hepaticojejunostomy. J Gastrointest Surg 2005; 9: 1163-1173

3 Emmett DS, Mallat DB. Double-balloon ERCP in patients who have undergone Roux-en-Y surgery: a case series. Gastrointest Endosc 2007; 66: $1038-1041$

4 Itoi $T$, Ishii $K$, Sofuni A et al. Single-balloon enteroscopy-assisted ERCP in patients with Billroth II gastrectomy or Roux-en-Y anastomosis (with video). Am J Gastroenterol 2010; 105: 93-99

5 Shimatani M, Matsushita M, Takaoka M et al. Effective "short" double-balloon enteroscope for diagnostic and therapeutic ERCP in patients with altered gastrointestinal anatomy: a large case series. Endoscopy 2009; 41: 849-854
Bibliography

DOI http://dx.doi.org/

10.1055/s-0042-105208

Endoscopy 2016; 48: E140-E141

(c) Georg Thieme Verlag KG

Stuttgart · New York

ISSN 0013-726X

\section{Corresponding author}

Kazumasa Nagai, MD

Center for Gastroenterology

Teine-Keijinkai Hospital

1-jo 12-chome

Maeda, Teine-ku

Sapporo 006-8555

Japan

Fax: +81-11-6852967

kazu4439@gmail.com 